\title{
Novel methylsulfonyl chalcones as potential antiproliferative drugs for human prostate cancer: Involvement of the intrinsic pathway of apoptosis
}

\author{
BASSEL ISMAIL $^{1}$, LAMIA GHEZALI ${ }^{1}$, ROKHAYA GUEYE ${ }^{2}$, YOUNESS LIMAMI ${ }^{1}$, CHRISTELLE POUGET $^{2}$, \\ DAVID Y. LEGER ${ }^{1}$, FREDERIQUE MARTIN ${ }^{1}$, JEAN-LOUIS BENEYTOUT ${ }^{1}$, JEAN-LUC DUROUX ${ }^{3}$, \\ MONA DIAB-ASSAF ${ }^{4}$, CATHERINE FAGNERE $^{2}$ and BERTRAND LIAGRE $^{1}$ \\ ${ }^{1}$ Biochemistry and Molecular Biology Laboratory, ${ }^{2}$ Organic Chemistry and Therapeutic Laboratory, \\ ${ }^{3}$ Biophysical Laboratory, Faculty of Pharmacy, University of Limoges, FR 3503 GEIST, EA1069, \\ GDR CNRS 3049, Limoges, France; ${ }^{4}$ Biochemistry Department, TM-PAC Team, \\ Faculty of Science - Section II, Lebanese University, Jdeidet, Lebanon
}

Received April 29, 2013; Accepted June 3, 2013

DOI: $10.3892 /$ ijo.2013.2024

\begin{abstract}
Limited success has been achieved in extending the survival of patients with metastatic and hormone-refractory prostate cancer (HRPC). There is a strong need for novel agents in the treatment and prevention of HRPC. In the present study, the apoptotic mechanism of action of RG003 (2'-hydroxy-4-methylsulfonylchalcone) and RG005 (4'-chloro2'-hydroxy-4-methylsulfonylchalcone) in association with intracellular signalling pathways was investigated in the hormone-independent prostate carcinoma cells PC-3 and DU145. We showed that these compounds induced apoptosis through the intrinsic pathway but not through the extrinsic one. We showed that synthetic chalcones induced an activation of caspase- 9 but not caspase- 8 in PC-3 cells. Even if both chalcones induced apoptosis in PC-3 cells, a dominant effect of RG003 treatment was observed resulting in a disruption of $\Delta \psi \mathrm{m}$, caspase- 9 and caspase- 3 activation, PARP cleavage and DNA fragmentation. Furthermore, in regard to our results, it is clear that the simultaneous inhibition of Akt and NF- $\mathrm{KB}$ signalling can significantly contribute to the anticancer effects of RG003 and RG005 in PC-3 prostate cancer cells. NF- $\mathrm{kB}$ inhibition was correlated with the reduction of COX-2 expression and induction of apoptosis. Our results clearly indicate for the first time that RG003 and RG005 exert their potent anti-proliferative and pro-apoptotic effects through the modu-
\end{abstract}

Correspondence to: Dr Bertrand Liagre, Biochemistry and Molecular Biology Laboratory, Faculty of Pharmacy, University of Limoges, FR 3503 GEIST, EA1069, GDR CNRS 3049, 2 rue du Docteur Marcland, 87025 Limoges Cedex, France

E-mail: bertrand.liagre@unilim.fr

Key words: chalcones, apoptosis, prostate cancer, cyclooxygenase-2, $\mathrm{NF}-\mathrm{kB}$ lation of $\mathrm{Akt} / \mathrm{NF}-\kappa \mathrm{B} / \mathrm{COX}-2$ signal transduction pathways in PC-3 prostate cancer cells with a dominant effect for RG003.

\section{Introduction}

Prostate cancer ( $\mathrm{PCa}$ ) is the most common malignant cancer in men and the second leading cause of cancer deaths (1). As in other tumor entities, curative therapy such as prostatectomy is limited to the organ-localized stage of PCa. PCa is initially responsive to hormonal therapy; however, in most cases, it becomes androgen-independent, evolving into more aggressive androgen refractory disease. At present, the treatment modality for patients with hormonal refractory $\mathrm{PCa}$ is chemotherapy (2), and this treatment is currently associated with significant side-effects and a reduced quality of life. Therefore, there is a need to develop novel approaches for this malignancy.

Chalcones are organic compounds that have an enone moiety between two aromatic rings. The development of these structures as potential drugs with biological properties has led to the discovery that these substances have antimalarial, anti-inflammatory and antitumor activities (3-5). Targeting apoptosis is a good strategy for cancer prevention and treatment, since many anticancer drugs induce apoptosis in cancer cells (6). Apoptosis occurs after a cascade of cell signalling that regulates proapoptotic and anti-apoptotic proteins by two major pathways: the mitochondrial and the death receptor pathways (7). The antitumor activity of chalcones is mostly related to modulation of the expression of proapoptotic and anti-apoptotic Bcl-2 family members and subsequent activation of the mitochondrial apoptotic pathway (8).

Several studies reported a role for arachidonic acid (AA) metabolism in many biological processes including cell proliferation, apoptosis and differentiation in many cancer types. Once released from phospholipid membranes, AA is converted into various prostanoids by cyclooxygenases (COX) (9). Two isoforms of COX, COX-1 and COX-2, have 
been identified: COX-1 has been purported to be a constitutive enzyme expressed in different tissues and maintains the physiological level of prostaglandins while modulating diverse cell processes such as cell proliferation, angiogenesis and platelet aggregation whereas COX-2 has been considered as inducible by different agents like proinflammatory cytokines, hormones and tumor promoters (10). COX-2 is overexpressed in some cancers, including PCa (11). Currently many compounds identified from plants were reported to inhibit cancer cell proliferation and COX-2 expression (12).

In the present study, apoptotic mechanism of RG003 (2'-hydroxy-4-methylsulfonylchalcone) and RG005 (4'-chloro-2'-hydroxy-4-methylsulfonylchalcone) (Fig. 1) in hormone-independent prostate carcinoma cells was investigated in association with COX-2 expression. Then, to understand the mechanisms implicated in the effect of RG003 and RG005, we studied intracellular signalling pathways.

\section{Materials and methods}

Cell lines, cell culture, treatment and light microscopy. The PC-3 and DU145 cell lines were purchased from the American Type Culture Collection (LGC Standards, Middlesex, UK). Cells were, respectively, seeded at $1.3 \times 10^{6}$ and $2 \times 10^{6}$ cells/well in $60-\mathrm{cm}^{2}$ tissue culture dishes and grown in RPMI-1640 medium supplemented with $10 \%$ fetal calf serum (FCS), $100 \mathrm{U} / \mathrm{ml}$ penicillin and $100 \mu \mathrm{g} / \mathrm{ml}$ streptomycin (all from Gibco-BRL, Cergy-Pontoise, France). Cultures were maintained in a humidified atmosphere with $5 \% \mathrm{CO}_{2}$ at $37^{\circ} \mathrm{C}$. Cells were grown for $24 \mathrm{~h}$ in culture medium prior to exposure or not to RG003 (2'-hydroxy-4-methylsulfonylchalcone) and RG005 (4'-chloro-2'-hydroxy-4-methylsulfonylchalcone), compounds synthesized by our research team. A stock solution of $10^{-2} \mathrm{M}$ RG003 and RG005 was prepared in dimethylsulfoxide (DMSO), and diluted in culture medium to give the appropriate final concentration. The same amount of vehicle (percentage of DMSO did not exceed $0.20 \%$ ) was added to control cells. Cell viability was determined by the trypan blue dye exclusion method. For light microscopy, after treatment, cultured cells were examined under phase-contrast microscopy (magnification, $\mathrm{x} 200$ ), and pictures were taken with an image acquisition system (Nikon, Champigny sur Marne, France).

Cell proliferation assay and viability assessment. Measurement of cell proliferation was determined using the 3-(4,5-dimethylthiazol-2-yl)-2,5-diphenyltetrazolium bromide (MTT) assay. PC-3 and DU145 cells were cultured and plated, respectively, at $5.6 \times 10^{3}$ and $8.5 \times 10^{3}$ cells/well in $10 \%$ FCS medium in 96-well culture plates and grown $24 \mathrm{~h}$ before treatment or not (time 0 ) with 5-40 $\mu \mathrm{M}$ RG003 and RG005 for 24-72 h. MTT tests were carried out daily as previously described (13) and experiments were performed in three independent assays.

PC-3 and DU145 cells were grown for $24 \mathrm{~h}$ then treated with RG003 and RG005 at 15 and $20 \mu \mathrm{M}$. After 24 and 48 h RG003 and RG005 treatment, cells were trypsinized and resuspended in complete medium. Each sample was mixed with trypan blue solution (0.14\% in HBSS). Colored (non-viable) and dye-excluding (viable) cells were counted on a Malassez hemocytometer. Controls were done with the same final DMSO concentration in the medium as samples.

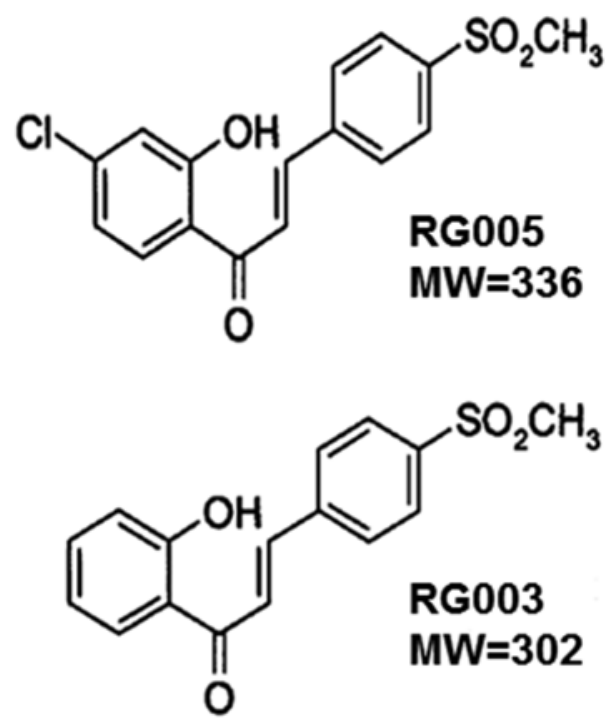

Figure 1. Chemical structures of RG003 (2'-hydroxy-4-methylsulfonylchalcone) and RG005 (4'-chloro-2'-hydroxy-4-methylsulfonylchalcone) MW, molecular weight.

Mitochondrial membrane potential $(\Delta \psi m)$. $\Delta \psi \mathrm{m}$ was estimated using 5,5',6,6'-tetrachloro-1,1',3,3'-tetraethylbenzimidazole carbocyanide iodide (JC-1, Molecular Probes). JC-1 is a fluorescent compound that exists as a monomer at low concentrations. At higher concentrations, it forms aggregates. Fluorescence of the JC-1 monomers is green, whereas that of aggregates is red. Mitochondria with intact membrane potential concentrate JC-1 into aggregates, which fluoresces red, whereas de-energized mitochondria cannot concentrate it and are stained green $(13,14)$.

PC-3 and DU145 cells were grown for $24 \mathrm{~h}$ before treatment with 15 and $20 \mu \mathrm{M}$ of RG003 and RG005, respectively, for 24 and $48 \mathrm{~h}$. Control cells were grown in medium containing the same amount of vehicle as treated cells. Then cells were incubated in $1 \mathrm{ml}$ of medium containing JC-1 $(1 \mu \mathrm{g} / \mathrm{ml})$ for $30 \mathrm{~min}$ at $37^{\circ} \mathrm{C}$ and pictures were taken with a fluorescence microscope.

Protein expression. After $15 \mu \mathrm{M}$ RG003 and RG005 treatment, PC-3 cells were washed and lysed in RIPA lysis buffer $(50 \mathrm{mM}$

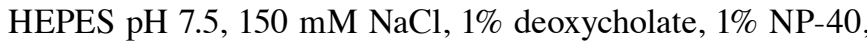
$0.1 \%$ SDS, $20 \mu \mathrm{g} / \mathrm{ml}$ aprotinin) containing protease inhibitors (Complete $^{\mathrm{TM}}$ Mini, Roche Diagnostics, Meylan, France). Briefly, as previously described (15), proteins (10-100 $\mu \mathrm{g})$ were separated by electrophoresis on SDS-polyacrylamide gels, transferred to PVDF membranes (Amersham Pharmacia Biotech, Saclay, France) and probed with respective human antibodiesagainstBax, caspase-8, caspase-9 and phospho-Akt (Ser 473) (Cell Signalling Technology, Ozyme, France), Bcl-2 and poly-ADP-ribose polymerase (PARP) (Santa Cruz Biotechnology, Tebu-Bio, Le Perray en Yvelines, France), and COX-2 (Cayman Chemical, Bertin Pharma, Montigny-le-Bretonneux, France). After incubation with secondary antibodies (Dako France SAS., Trappes, France), blots were developed using the ECL Plus Western Blotting Detection System (Amersham Pharmacia Biotech) and G:BOX system (Syngene, Ozyme, Saint Quentin en Yvelines, France). Membranes were then reblotted with anti- $\beta$-actin (Sigma Aldrich, Saint Quentin Fallavier, France) used as a loading control. 
PC-3 cells
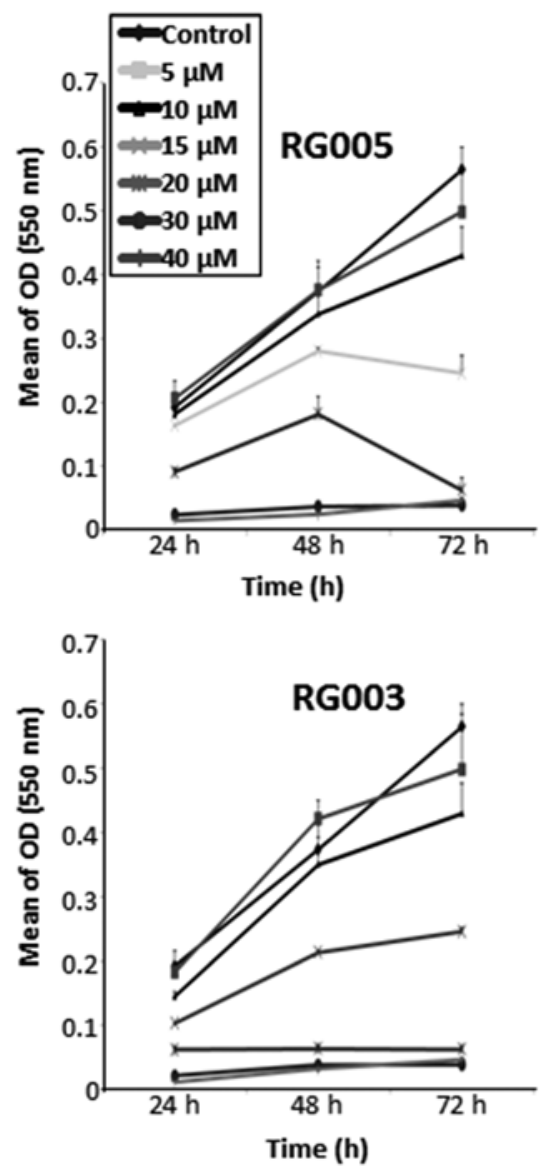

DU145 cells
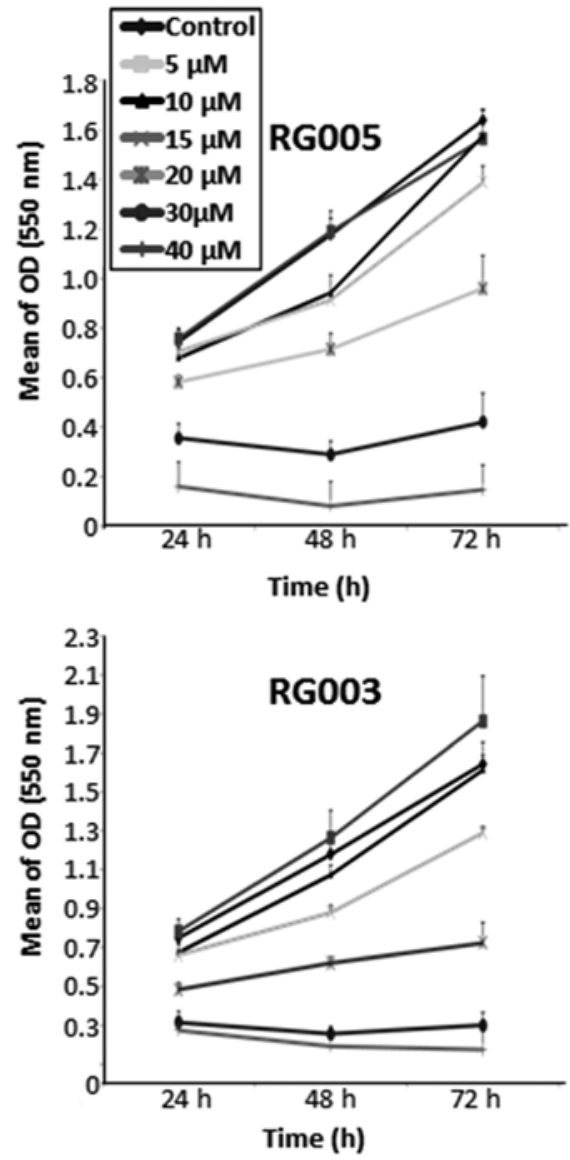

Figure 2. Effect of RG005 and RG003 on PC-3 and DU145 cell growth. Cells were cultured in 10\% FCS medium during $24 \mathrm{~h}$ and treated or not with 5-40 $\mu \mathrm{M}$ RG005 or RG003 for 24-72 h. Cell proliferation was followed by MTT test.

Caspase-3 activity. Caspase-3 activity was assayed using Quantikine $^{\circledR}$ human active caspase-3 (R\&D Systems) as previously described (16). PC-3 cells were treated or not with $15 \mu \mathrm{M}$ of RG003 and RG005 for 24 and $48 \mathrm{~h}$, and then incubated with $10 \mu \mathrm{M}$ biotin-ZVKD-fmk inhibitor for $1 \mathrm{~h}$ at $37^{\circ} \mathrm{C}$. Caspase- 3 activity was measured in accordance with the manufacturer's protocol (R\&D Systems). Briefly, cells were harvested, washed in PBS and resuspended in extraction buffer containing protease inhibitors. Standards and sample extracts containing covalently linked active caspase-3-ZVKD-biotin were added to a microplate pre-coated with monoclonal antibody specific for caspase-3. Then, streptavidin conjugated to horseradish peroxidase was added to the wells. The amount of active caspase- 3 was quantified by colorimetry at $450 \mathrm{~nm}$ after addition of HRP substrate.

Apoptosis quantification: DNA fragmentation. PC-3 cells were seeded at $1.3 \times 10^{6}$ cells in $60-\mathrm{cm}^{2}$ tissue culture dishes and then treated or not with $15 \mu \mathrm{M}$ of RG003 and RG005 for 24 and $48 \mathrm{~h}$. Apoptosis was quantified on pooled cells (floating and adherent) using 'cell death' enzyme-linked immunosorbent assay (ELISA) (Cell Death Detection ELISA ${ }^{\text {PLUS }}$, Roche Diagnostics). Cytosol extracts were obtained according to the manufacturer's protocol and apoptosis was measured as previously described (17).
Subcellular protein fractionation. PC-3 cells were incubated alone or with $15 \mu \mathrm{M}$ of RG003 and RG005 for 24 and $48 \mathrm{~h}$. Cytosolic and nuclear fractions were obtained using the Subcellular Protein Fractionation Kit according to the manufacturer's protocol (Thermo Fischer Scientific, Rockford, IL, USA) as previously described (13).

Electromobility shift assay (EMSA). EMSA experiments were performed using DIG Gel Shift kit (Roche Diagnostics) (18). Briefly, nuclear extracts were prepared from PC-3 cells treated or not with $15 \mu \mathrm{M}$ of RG003 and RG005 for 24 and $48 \mathrm{~h}$. NF- $\mathrm{KB}$ binding reactions were carried out with $10 \mu \mathrm{g}$ nuclear proteins incubated with digoxigenin (DIG) labeled $\mathrm{NF}-\kappa \mathrm{B}$ probe according to the manufacturer's protocol. The samples were loaded on a $5 \%$ native polyacrylamide gel in Tris-Borate-EDTA buffer. After transfer to nylon membranes and incubation with anti-DIG antibody conjugated with alkaline phosphatase, gel mobility shift was visualized by incubation with $\mathrm{CSPD}^{\circledR}$ chemiluminescence reagent and G:BOX system (Syngene, Ozyme, Saint Quentin en Yvelines, France). Quantification of each band was performed by densitometry analysis software in respect of band intensity and band area. Results are expressed relative to controls in arbitrary units. 
A

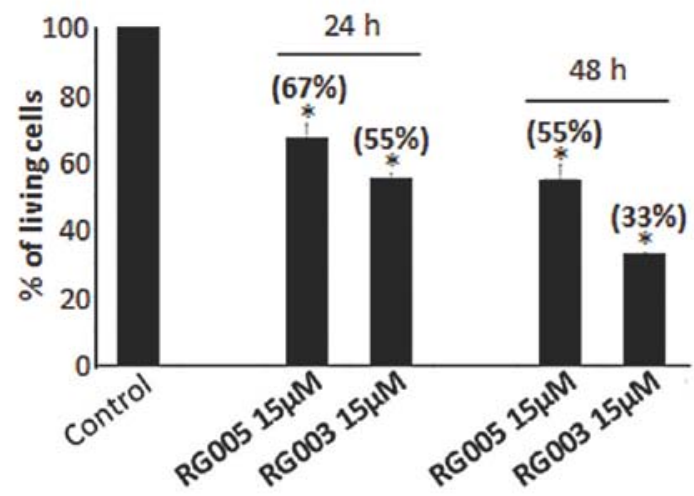

DU145 cells

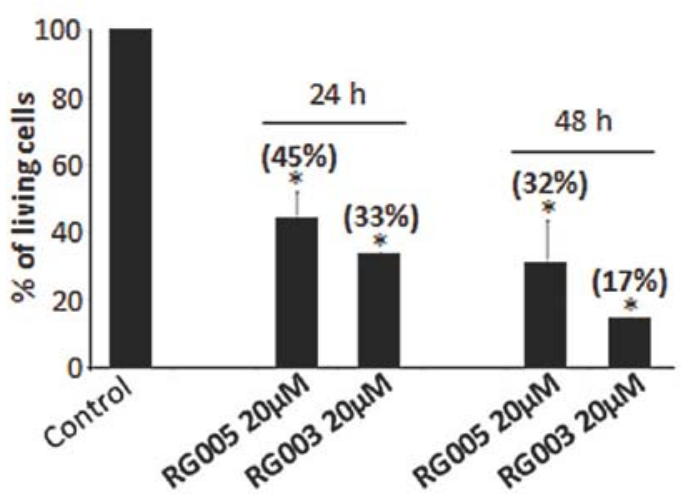

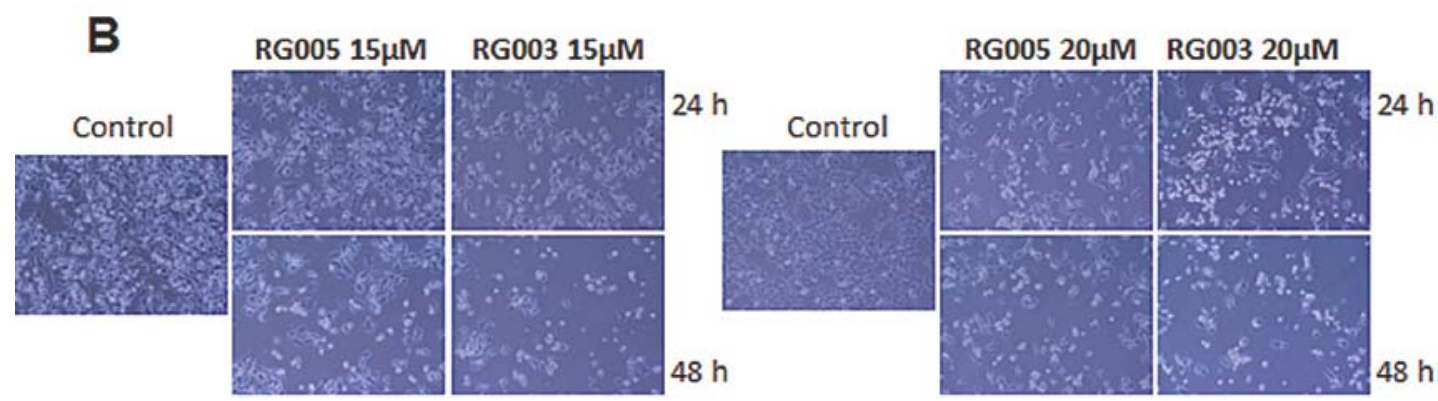

Figure 3. Effect of RG005 and RG003 on PC-3 and DU145 cell viability. PC-3 and DU145 cells were grown for 24 h then treated with RG003 and RG005 at 15 or $20 \mu \mathrm{M}$ for 24 and $48 \mathrm{~h}$. (A) Relative cell viability was assessed by trypan blue dye exclusion method. Results are presented as percentage of living cells compared to control $\left(\mathrm{n}=3,{ }^{*} \mathrm{p}<0.05\right)$. (B) Morphologic changes in prostate cancer cells. Original magnification, $\mathrm{x} 200$.

Statistical analysis. Data are expressed as the arithmetic means \pm standard deviation (SD) of separate experiments. The statistical significance of results obtained from in vitro studies was evaluated by the two tailed unpaired Student's t-test, with $\mathrm{p}<0.05$ being considered as significant.

\section{Results}

Chalcone effect on PC-3 and DU145 cell proliferation and morphological modifications. Cells were cultured in $10 \%$ FCS-medium with or without chalcones $(5-40 \mu \mathrm{M})$ for 24-72 $\mathrm{h}$ and cell proliferation was evaluated by the MTT test (Fig. 2). Under our experimental conditions, a decrease in proliferation was observed as early as $24 \mathrm{~h}$ after chalcone treatment in a dose- and time-dependent manner. To confirm these results, relative cell viability was assessed by trypan blue dye exclusion with, respectively, 15 and $20 \mu \mathrm{M}$ chalcone treatment for PC-3 and DU145 cells (Fig. 3A). Trypan blue dye proved the antiproliferative effect of the two chalcones by decreasing percentage of living cells with significative dominant effect for RG003 treatment in both cell lines (33\% of living cells versus $55 \%$ for RG003 and RG005, respectively, for PC-3 cells after 48 -h treatment, $\mathrm{p}<0.05 ; 17 \%$ versus $32 \%$ for RG003 and RG005, respectively, for DU145 cells after 48 -h treatment, $\mathrm{p}<0.05$ ). For following experiments, we used both compounds at 15 and $20 \mu \mathrm{M}$ for PC-3 and DU145 cells, respectively.

Direct observation with phase-contrast microscopy demonstrated that cells treated with compounds showed numerous morphological differences compared to control cells (Fig. 3B). Indeed, cell shrinkage, cytoplasm condensation and formation of cytoplasmic filaments appeared after compound treatment. Furthermore, the pictures of treated cells confirmed that antiproliferative effect was more important with RG003 than RG005 treatment.

Chalcone induces disruption of $\Delta \psi m$ in PC-3 and DU145 cell lines. To determine potential mechanisms by which chalcone inhibited the human PC-3 and DU145 cell proliferation, we analyzed the effect of RG003 and RG005 on $\Delta \psi \mathrm{m}$, because alterations in mitochondrial structure and function have been shown to play a crucial role at early stages of apoptosis (19).

$\Delta \psi \mathrm{m}$ was analyzed after 24 and $48-\mathrm{h}$ treatment with chalcones using JC-1. We found that compounds decreased $\Delta \psi \mathrm{m}$ in both PC-3 and DU145 cells in a time-dependent manner (Fig. 4), shown by the incorporation of JC-1 monomers into mitochondria (green fluorescence), compared with cytosolic JC-1 aggregate formation at high membrane potentials in control cells (red fluorescence). Furthermore, this assay showed a dominant effect of RG003 on early intrinsic apoptosis compared to RG005.

Chalcones induce apoptosis with activation of intrinsic pathway. The process of apoptosis is tightly regulated by a large variety of proteins that promote or block cell death at different stages of apoptosis. The regulatory role of $\mathrm{Bcl}-2$ family proteins is now well established. The $\mathrm{Bax} / \mathrm{Bcl}-2$ ratio, which is a critical determinant of apoptosis (20), was deter- 

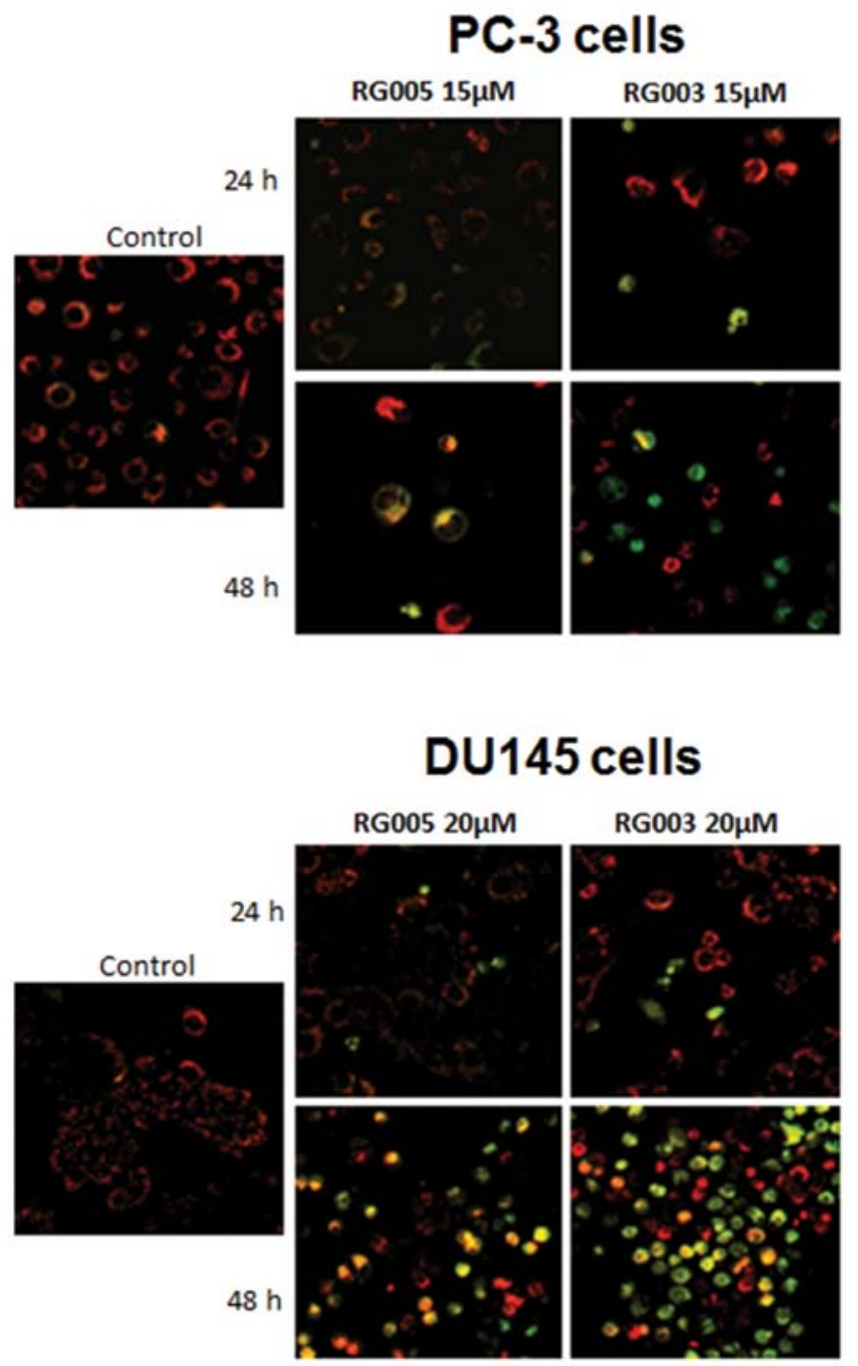

Figure 4. Effect of RG005 and RG003 on PC-3 and DU145 cell mitochondrial membrane potential. Cells were cultured in $10 \%$ FCS medium for $24 \mathrm{~h}$ and treated or not with RG003 or RG005 at 15 or $20 \mu \mathrm{M}$ for 24 and $48 \mathrm{~h}$, respectively. After treatment, cells were incubated with medium containing JC-1 $(1 \mu \mathrm{g} / \mathrm{ml})$ for $30 \mathrm{~min}$ at $37^{\circ} \mathrm{C}$. Red fluorescence represents mitochondria with intact membrane potential whereas green fluorescence represents de-energized mitochondria. Pictures were taken with a Nikon microscope Eclipse E800 (x100). The pictures are representative of three separate experiments.

mined after western blot analysis. Apoptotic ratio (Bax/Bcl-2) obtained with western blot analysis indicated that after $24 \mathrm{~h}$ $15 \mu \mathrm{M}$ RG003 treatment increased the apoptotic ratio (1.5-fold versus control, $\left.{ }^{*} \mathrm{p}<0.05\right)$ whereas RG005 treatment had no effect on PC-3 cells (Fig. 5A). After 48-h treatment, RG003 and RG005 both increased the Bax/Bcl-2 ratio (2.4- and 1.6-fold, respectively, versus control, ${ }^{* *} \mathrm{p}<0.01$ and $\left.{ }^{*} \mathrm{p}<0.05\right)$ and our results showed again a dominant effect of RG003 treatment. In fact, the increase in the Bax/Bcl-2 ratio seems to be mainly due to a decline in Bcl-2 expression during RG003 and RG005 chalcone treatment.

Apoptosis induction requires activation of specific proteins. It is well known that apoptosis is characterized by chromatin condensation and DNA fragmentation, and is mediated by the cysteine protease family called caspases. Caspase activation can be regulated through an extrinsic or intrinsic signalling pathway. The extrinsic pathway, which involves
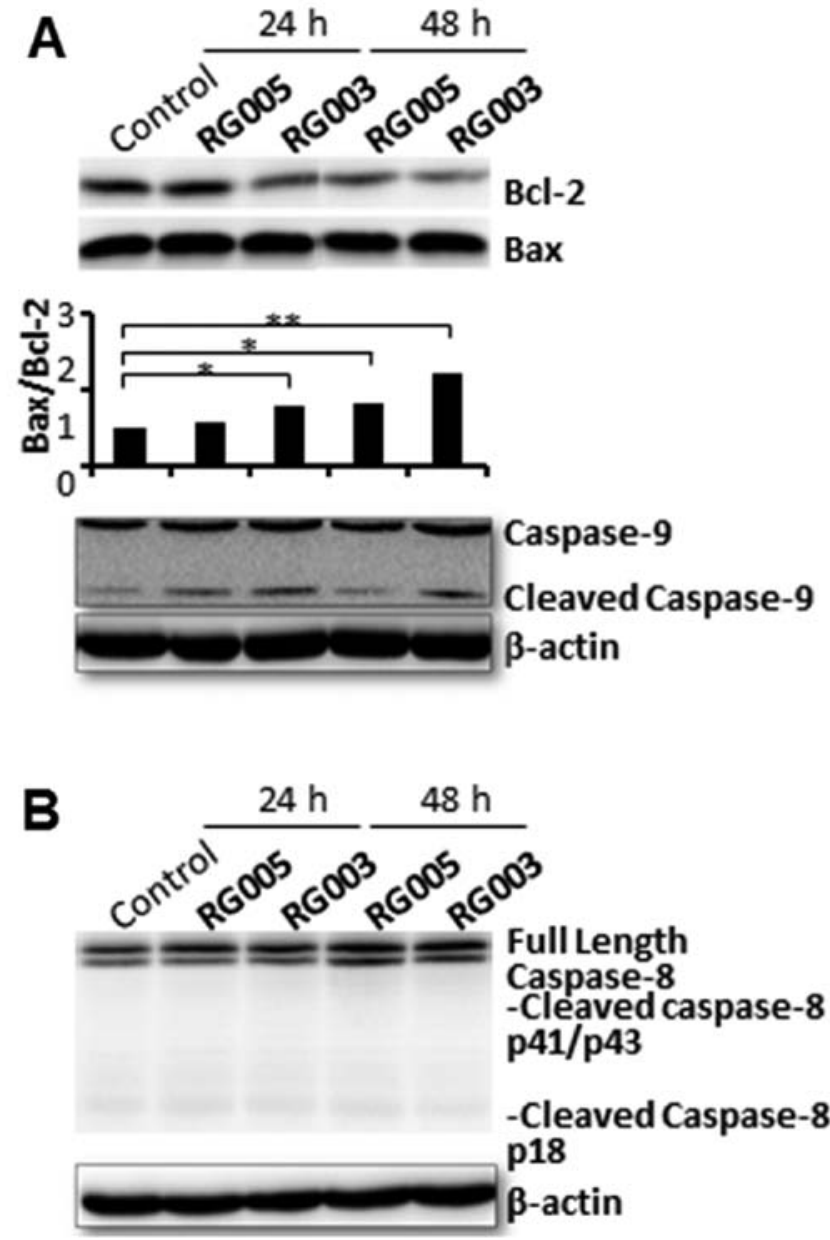

Figure 5. Effect of RG005 and RG003 on (A) Bcl-2, Bax, caspase-9 and (B) caspase-9 expression in PC-3 cells. Cells were cultured in 10\% FCS medium for $24 \mathrm{~h}$ and treated or not with $15 \mu \mathrm{M}$ RG003 or RG005 for 24 and $48 \mathrm{~h}$, respectively. Protein expression was analyzed by western blotting. $\beta$-actin was used as a loading control. Blots are representative of three separate experiments. Results of $\mathrm{Bax} / \mathrm{Bcl}-2$ ratio are presented as $\mathrm{n}$-fold increase compared to control $\left({ }^{*} \mathrm{p}<0.05,{ }^{* *} \mathrm{p}<0.01\right)$.

Fas and tumor necrosis factor receptor stimulation, activates caspase-8. The intrinsic pathway, which may be the primary means of activating apoptotic caspase in mammals, triggers the mitochondrial release of cytochrome $c$, which oligomerizes with Apaf-1 and procaspase-9 to form the apoptosome complex. Activated caspase-9 in this complex activates caspase-3 which is the major executioner of apoptosis (21). PARP is one of the best known caspase substrates and its inactivation by cleavage is now an apoptosis hallmark. DNA fragmentation occurs simultaneously with this phenomenon and is now considered as a major marker of apoptotic cells.

In our study, we showed that intrinsic apoptosis pathway was implicated in contrast to extrinsic apoptosis pathway. Indeed, we showed that chalcones induced an activation of caspase-9 but not caspase-8 in PC-3 cells as shown in Fig. 5A and B, respectively. RG005 and RG003 induced a cleavage of caspase- 9 at 24 and $48 \mathrm{~h}$ but the expression of cleaved fragment of caspase- 9 after RG003 treatment is more important than RG005 treatment. Consequently, RG005 induced only a slight activation of executive caspase- 3 activity $(+1.2$-fold 

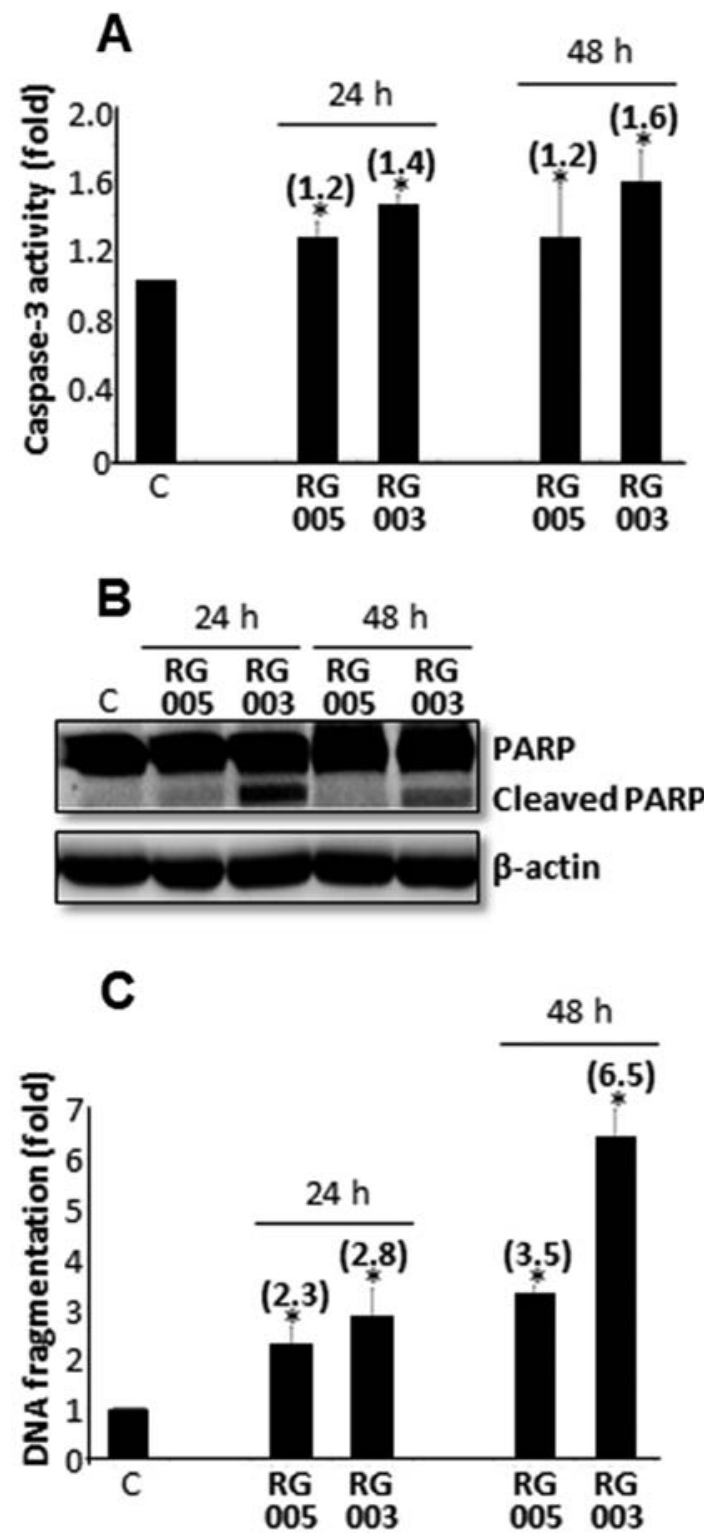

Figure 6. Effect of RG005 and RG003 on caspase-3 activity, PARP cleavage and DNA fragmentation in PC-3 cells. Cells were cultured in 10\% FCS medium for $24 \mathrm{~h}$ and treated or not with $15 \mu \mathrm{M}$ RG003 or RG005 for 24 and $48 \mathrm{~h}$, respectively. (A) Caspase-3 activity was assayed using Quantikine human active caspase-3 (R\&D Systems). Following treatment, cells were incubated with $10 \mu \mathrm{M}$ biotin-ZVKD-fmk inhibitor for $1 \mathrm{~h}$ at $37^{\circ} \mathrm{C}$. The amount of active caspase-3 was quantified by colorimetry at $450 \mathrm{~nm}$; values are expressed as mean $\pm \mathrm{SD}$ of three separate experiments and reported as $\mathrm{n}$-fold $\left({ }^{*} \mathrm{p}<0.05\right)$ compared to control. (B) PARP cleavage was analyzed by western blotting. PARP cleavage was assessed by detection of a $85 \mathrm{kDa}$ band (cleaved PARP) after probing with a mouse anti-human PARP antibody. $\beta$-actin was used as a loading control. Blots are representative of three separate experiments. (C) DNA fragmentation was quantified from cytosol extracts according to the manufacturer's instructions (Cell Death Detection ELISAplus, Roche Diagnostics). Results were reported as $\mathrm{n}$-fold compared to control. Values are expressed as mean $\pm \mathrm{SD}$ ( $\mathrm{p}$-value relative to control group, ${ }^{*} \mathrm{p}<0.05$ ).

versus control at 24 and $48 \mathrm{~h}, \mathrm{p}<0.05)$. On the contrary, caspase-3 activity was greater after RG003 treatment $(+1.4-$ and +1.6 -fold at 24 and $48 \mathrm{~h}$, respectively, versus control, $\mathrm{p}<0.05$ ) (Fig. 6A). These observations were directly correlated with PARP cleavage because western blot analysis detected the native form of PARP but not a significant cleaved fragment in RG005 treated PC-3 cells. In contrast, cleaved fragment of PARP was strongly expressed starting at 24-h RG003 treatment and maintained after 48-h treatment (Fig. 6B).

DNA fragmentation, considered as a major marker of apoptotic cells, was observed in PC-3 cells after chalcone treatment. Quantitative determination of cytoplasmic histone-associatedDNA-fragments (mono and oligonucleosomes) was performed by ELISA in our study. Results showed that DNA fragmentation was induced in PC-3 cells after 24-h RG005 and RG003 treatment (+2.3- and +2.8-fold, respectively, versus control, $\mathrm{p}<0.05)$ whereas DNA fragmentation was markedly enhanced after 48-h RG003 treatment compared to RG005 tretament (+6.5and +3.5 -fold, respectively, versus control, p<0.05) (Fig. 6C).

In summary, even if both chalcones induced apoptosis of PC-3 cells, a dominant effect of RG003 treatment was observed resulting in activation of the intrinsic pathway with disruption of $\Delta \psi \mathrm{m}$, caspase- 9 and caspase-3 activation, PARP cleavage and DNA fragmentation.

Downregulation of survival pathways after chalcone treatment in PC-3 cells. Nuclear factor $-\kappa \mathrm{B}(\mathrm{NF}-\kappa \mathrm{B})$ is a ubiquitous transcription factor that has been shown to promote cell survival by initiating the transcription of genes involved in cell proliferation or encoding anti-apoptotic proteins (22). Akt promotes cell survival by phosphorylating substrates that decrease the activity of pro-apoptotic proteins or increase the activity of anti-apoptotic proteins (23). We analyzed the effect of RG005 and RG003 on two survival pathways: Akt and NF- $\kappa$ B. Western blot analysis showed that both chalcone markedly inhibited Akt phosphorylation in PC-3 cells (Fig. 7A). Since NF- $\kappa$ B activation is critical for apoptosis resistance, we examined the effect of RG005 and RG003 on nuclear activation of NF- $\kappa \mathrm{B}$. Our results showed that chalcone treatment inhibited NF- $\kappa \mathrm{B}$ activation. Furthermore, this inhibition was stronger with RG003 treatment (Fig. 7B).

Effect of RG005 and RG003 on COX-2 expression in PC-3 cells. It is well known that COX-2 expression is correlated with the activities of intracellular signalling proteins such as $\mathrm{NF}-\kappa \mathrm{B}$ (24). Furthermore, we showed recently that COX-2 positively regulated Akt signalling and enhanced survival of cancer cells exposed to anticancer agents (17). Numerous studies have shown that COX-2 expression prevents apoptosis in cancer cells, especially in colon (25) and prostate cancer $(26,27)$. Here, we showed that both chalcones reduced significantly expression of COX-2 by 24-h treatment in PC-3 cells. RG003 was the more effective in decreasing COX-2 expression (Fig. 8).

\section{Discussion}

In light of the reported chemopreventive and chemosensitive effects of chalcones on various tumor cells and animal models, we postulated that our new methylsulfonyl chalcones may mediate their effects through apoptosis induction with suppression of cell survival pathways. Here, we observed that RG003 and RG005 could indeed suppress Akt/NF- $\mathrm{B} / \mathrm{COX}-2$ activation and exert significant anti-proliferative and apoptotic effects in androgen-independent PCa cells. We also clearly demonstrated that RG003 and RG005 induce apoptosis through mitochondrial pathway in human PCa PC-3 cells. 

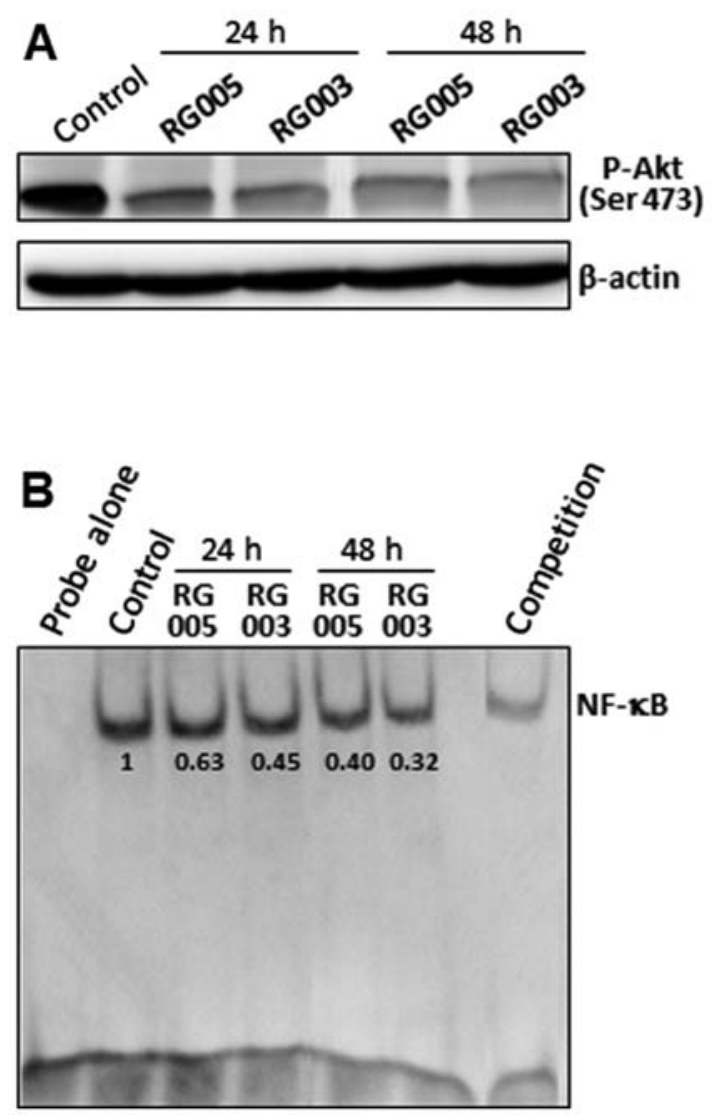

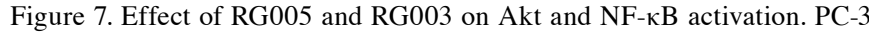
cells were cultured in $10 \%$ FCS medium for $24 \mathrm{~h}$ and treated or not with $15 \mu \mathrm{M}$ RG003 or RG005 for 24 and $48 \mathrm{~h}$, respectively. (A) Akt phosphorylation was determined by western blot analysis. $\beta$-actin was used as a loading control. Blots are representative of three separate experiments. (B) EMSA experiments were performed using DIG Gel Shift Kit (Roche Diagnostics) on nuclear extracts. Blots are representative of three separate experiments.

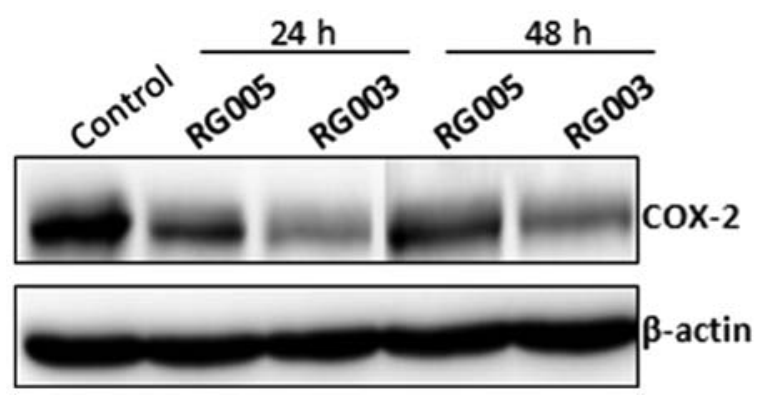

Figure 8. Effect of RG005 and RG003 on COX-2 expression. PC-3 cells were cultured in $10 \%$ FCS medium for $24 \mathrm{~h}$ and treated or not with $15 \mu \mathrm{M}$ RG003 or RG005 for 24 and $48 \mathrm{~h}$, respectively. COX-2 protein expression was determined by western blot analysis. $\beta$-actin was used as a loading control. Blots are representative of three separate experiments.

$\mathrm{PCa}$ is very uncommon in men younger than 45 years, but becomes more common with advancing age (28). Many of the risk factors for prostate cancer are more prevalent in the developed world, including longer life expectancy, alcohol/tobacco intake, and diets high in red meat (29). Interestingly, the American Dietetic Association and Dieticians of Canada report a decreased risk of developing prostate cancer for those following a vegetarian diet (30). However, the specific causes of prostate cancer still remain unknown (31).

Researchers have established a few PCa cell lines to investigate the mechanism involved in the progression of PCa. Androgen-independent PC-3 and DU145 cells are commonly used. Although previous studies have shown that chalcones can inhibit the cell proliferation in these human prostate cancer cells including PC-3 (32-34) and DU145 $(33,35)$ cells, our study is the first report on the specific examination of intrinsic apoptosis and $\mathrm{Akt} / \mathrm{NF}-\kappa \mathrm{B} / \mathrm{COX}-2$ pathways in human $\mathrm{PCa}$ cells upon synthetic chalcones exposure.

Apoptosis is characterized by chromatin condensation and DNA fragmentation, and is mediated by caspases (36). Mitochondria are involved in a variety of key events, including release of caspase activators, changes in electron transport, loss of mitochondrial membrane potential $(\Delta \psi \mathrm{m})$, and participation of both pro- and anti-apoptotic Bcl-2 family proteins (37). Alterations in mitochondrial structure and function have been shown to play a crucial role in caspase-9-dependent apoptosis (38). Caspase-9 cleaves and activates caspase-3, the executioner caspase, which cleaves PARP and activates endonucleases leading to DNA fragmentation (38). To analyze the effect of RG003 and RG005 on induction of apoptosis in PCa cells, apoptosis was evaluated on pooled cell fractions (floating and adherent). Mitochondria have, apart from their function in respiration, an important role in the apoptotic-signalling pathway. It is well known that the modification of $\Delta \psi \mathrm{m}$ depends on the nature of the stimulus and the cell system and the collapse of $\Delta \psi \mathrm{m}$ is an early step in the apoptotic cascade (39). We showed that RG003 and RG005 decreased $\Delta \psi \mathrm{m}$ in both PC-3 and DU145 cells in a time-dependent manner.

Moreover, the $\mathrm{Bax} / \mathrm{Bcl}-2$ ratio, which is a critical determinant of apoptosis (20), was determined after western blot analysis. In our study, the increase in the $\mathrm{Bax} / \mathrm{Bcl}-2$ ratio seems to be mainly due to a decline in Bcl-2 expression during RG003 and RG005 chalcone treatment, with a dominant effect of RG003 treatment. Since Bcl-2 protects human prostate cancer cells from the induction of apoptosis (40), its downregulation could contribute to the capability of RG003 and RG005 to induce apoptosis in PCa PC-3 cells.

Caspase-3 is a key executioner of apoptosis, its activation is mediated by the initiator caspases such as caspase- 8 and caspase-9 (41). We showed that the intrinsic apoptosis pathway was implicated but not the extrinsic apoptosis pathway. We showed that chalcones induced activation of caspase- 9 but not caspase- 8 in PC-3 cells. Consequently, RG005 induced only a slight activation of executive caspase-3 activity compared to RG003 treatment. These observations were directly correlated with PARP cleavage because western blot analysis detected the native form of PARP but not the significant cleaved fragment in RG005 treated PC-3 cells. In contrast, cleaved fragment of PARP was strongly detected with RG003 treatment. PARP is a nuclear enzyme involved in the repair of DNA damage (42). Moreover, it is known that PARP is a substrate for caspases such as caspase-3 and is typically cleaved and inactivated during the apoptotic process (43). DNA fragmentation occurs simultaneously with this phenomenon and is considered as a major marker of apoptotic cells. Apoptosis quantification was performed by ELISA, and results showed that DNA fragmentation was induced 
in PCa PC-3 cells by 24-h chalcone treatment. In our study, chalcone treatment in PCa PC-3 cells induces a disruption of $\Delta \psi \mathrm{m}$, caspase-9 and -3 activation, PARP cleavage (only after RG003 treatment) and DNA fragmentation. Furthermore, we demonstrated a dominant effect of RG003 treatment versus RG005 one.

Among the cell signalling pathways that promote cell survival, Akt is one of the most important (44). It has been previously reported that the level of Akt activation is drastically enhanced in androgen-independent PC-3 cells as compared with the androgen-dependent cells (45). Activated Akt can also exert anti-apoptotic effects, positively regulate NF- $\mathrm{B}$ transcription, modulate angiogenesis, promote tumor invasion/metastasis and antagonize cell cycle arrest (46). Hence, in the present report, we investigated the effects of RG003 and RG005 on Akt pathway in androgen-independent PCa PC-3 cells. We found that chalcones inhibited the expression of phospho-Akt (Ser 473) in PC-3 cells. Akt is also reported to modulate the $\mathrm{NF}-\kappa \mathrm{B}$ transcription factor through the phosphorylation of p65 to enhance the transcriptional activity of NF- $\kappa \mathrm{B}(47)$. $\mathrm{NF}-\kappa \mathrm{B}$ activation is also known to regulate the expression of various cell survival, proliferative, metastatic and angiogenic gene products (48). Our results showed that RG003 and RG005 treatment inhibited $\mathrm{NF}-\kappa \mathrm{B}$ activation. In regard to these results, it is clear that the simultaneous inhibition of Akt and $N F-\kappa B$ signalling can significantly contribute to the anticancer effects of RG003 and RG005 in PCa PC-3 cells.

Recent studies on the relationship between the arachidonic acid (AA) cascade and carcinogenesis revealed novel molecular targets for cancer treatment (49). It has been demonstrated that the metabolism of AA, a polyunsatured fatty acid, by either the COX or lipoxygenase (LOX) pathway, generates a host of proinflammatory metabolites known to modulate diverse physiological and pathological responses such as angiogenesis, apoptosis and hyperproliferation $(50,51)$. COX-2 has been demonstrated to play an important role in apoptosis resistance and carcinogenesis, particularly in colon carcinogenesis $(25,52)$. Previous studies from our laboratory reported a role for COX-2 in resistance to apoptosis in colorectal and prostate cancer cells $(53,54)$. Here, we demonstrated that RG003 and RG005 reduced COX-2 expression in PCa PC-3 cells. We demonstrated that $\mathrm{NF}-\kappa \mathrm{B}$ activation was inhibited after chalcone treatment. Its inhibition was correlated with reduction of COX-2 expression and induction of apoptosis. $\mathrm{NF}-\kappa \mathrm{B}$ activation signalling pathway was reported to regulate COX-2 expression and to promote cell survival (24).

Our results clearly indicate for the first time that RG003 and RG005 exert their potent anti-proliferative and pro-apoptotic effects through the modulation of $\mathrm{Akt} / \mathrm{NF}-\kappa \mathrm{B} / \mathrm{COX}-2$ signal transduction pathways in PCa PC-3 cells and do not act specifically on any one cellular signalling cascade. It is obvious that RG003 and RG005 are not active against a specific signalling cascade but they can interfere with a multitude of targets in PCa PC-3 cells. This is quite relevant to the changing paradigm in cancer therapy, as increasing evidence indicates that the mono-targeted drugs, once called smart drugs, have not had a significant impact on cancer treatment and the use of multi-targeted drugs has become increasingly accepted, as it is obvious that cancer is caused by dysregulation of multiple pathways (55).
Our results show that RG003 has a dominant effect compared to RG005 treatment on apoptosis of PCa cells. The only structural difference is the presence of one chlorine atom in the A cycle at 4' position for RG005. To understand the difference of their biological activities, two major characteristics could be implicated: spatial conformation and redox reactivity. Both characteristics can be examined by molecular modeling as previously used by our team (56). Furthermore, the significance of our in vitro study between RG003 and RG005 effects in PCa cells is very encouraging suggesting the relevance of testing these compounds in xenograft animal models.

\section{Acknowledgements}

The authors are grateful to Claire Carrion for help in performing mitochondrial membrane potential assays. This research was supported by grants from the French Ministry of Education and Research and from the Lebanese National Council for Scientific Research (CNRS-L, doctoral scholarship to B.I.).

\section{References}

1. Jemal A, Siegel R, Ward E, Hao Y, Xu J and Thun MJ: Cancer statistics, 2009. CA Cancer J Clin 59: 225-249, 2009.

2. Dreicer R: Current status of cytotoxic chemotherapy in patients with metastatic prostate cancer. Urol Oncol 26: 426-429, 2008.

3. Alcaraz MJ, Vicente AM, Araico A, Dominguez JN, Terencio MC and Ferrándiz ML: Role of nuclear factor-kappaB and heme oxygenase- 1 in the mechanism of action of an antiinflammatory chalcone derivative in RAW 264.7 cells. Br J Pharmacol 142: 1191-1199, 2004.

4. Domínguez JN, León C, Rodrigues J, Gamboa de Domínguez N, Gut J and Rosenthal PJ: Synthesis and evaluation of new antimalarial phenylurenyl chalcone derivatives. J Med Chem 48: 3654-3658, 2005.

5. Liu X and Go ML: Antiproliferative activity of chalcones with basic functionalities. Bioorg Med Chem 15: 7021-7034, 2007.

6. Aggarwal BB and Shishodia S: Molecular targets of dietary agents for prevention and therapy of cancer. Biochem Pharmacol 71: 1397-1421, 2006

7. $\mathrm{Hu} \mathrm{W}$ and Kavanagh JJ: Anticancer therapy targeting the apoptotic pathway. Lancet Oncol 4: 721-729, 2003.

8. Shen KH, Chang JK, Hsu YL and Kuo PL: Chalcone arrests cell cycle progression and induces apoptosis through induction of mitochondrial pathway and inhibition of nuclear factor kappa $B$ signalling in human bladder cancer cells. Basic Clin Pharmacol Toxicol 101: 254-261, 2007.

9. Smith WL, DeWitt DL and Garavito RM: Cyclooxygenases: structural, cellular, and molecular biology. Annu Rev Biochem 69: 145-182, 2000.

10. Xie WL, Chipman JG, Robertson DL, Erikson RL and Simmons DL: Expression of a mitogen-responsive gene encoding prostaglandin synthase is regulated by mRNA splicing. Proc Natl Acad Sci USA 88: 2692-2696, 1991.

11. Gupta S, Srivastava M, Ahmad N, Bostwick DG and Mukhtar H: Over-expression of cyclooxygenase-2 in human prostate adenocarcinoma. Prostate 42: 73-78, 2000.

12. Wenzel U, Kuntz S, Brendel MD and Daniel H: Dietary flavone is a potent apoptosis inducer in human colon carcinoma cells. Cancer Res 60: 3823-3831, 2000.

13. Corbiere C, Liagre B, Terro F and Beneytout JL: Induction of antiproliferative effect by diosgenin through activation of $\mathrm{p} 53$, release of apoptosis-inducing factor (AIF) and modulation of caspase-3 activity in different human cancer cells. Cell Res 14: 188-196, 2004.

14. Smiley ST, Reers M, Mottola-Hartshorn C, et al: Intracellular heterogeneity in mitochondrial membrane potentials revealed by a J-aggregate-forming lipophilic cation JC-1. Proc Natl Acad Sci USA 88: 3671-3675, 1991.

15. Lepage C, Liagre B, Cook-Moreau J, Pinon A and Beneytout JL: Cyclooxygenase-2 and 5-lipoxygenase pathways in diosgenin-induced apoptosis in HT-29 and HCT-116 colon cancer cells. Int J Oncol 36: 1183-1191, 2010. 
16. Leger DY, Liagre B and Beneytout JL: Role of MAPKs and NF- $\kappa \mathrm{B}$ in diosgenin-induced megakaryocytic differentiation and subsequent apoptosis in HEL cells. Int J Oncol 28: 201-207, 2006.

17. Bertrand J, Liagre B, Ghezali L, Beneytout JL and Leger DY: Cyclooxygenase-2 positively regulates Akt signalling and enhances survival of erythroleukemia cells exposed to anticancer agents. Apoptosis 18: 836-850, 2013.

18. Ghezali L, Leger DY, Limami Y, Cook-Moreau J, Beneytout JL and Liagre B: Cyclopamine and jervine induce COX-2 overexpression in human erythroleukemia cells but only cyclopamine has a pro-apoptotic effect. Exp Cell Res 319: 1043-1053, 2013.

19. Green DR and Reed JC: Mitochondria and apoptosis. Science 281: 1309-1312, 1998

20. Oltvai ZN, Milliman CL and Korsmeyer SJ: Bcl-2 heterodimerizes in vivo with a conserved homolog, Bax, that accelerates programmed cell death. Cell 74: 609-619, 1993.

21. MacKenzie SH, Schipper JL and Clark AC: The potential for caspases in drug discovery. Curr Opin Drug Discov Dev 13 568-576, 2010.

22. Liu YQ, Hu XY, Lu T, Cheng YN, Young CY, Yuan HQ and Lou HX: Retigeric acid B exhibits antitumor activity through suppression of nuclear factor- $\kappa \mathrm{B}$ signalling in prostate cancer cells in vitro and in vivo. PLoS One 7: e38000, 2012.

23. Datta SR, Dudek H, Tao X, Masters S, Fu H, Gotoh Y and Greenberg ME: Akt phosphorylation of BAD couples survival signals to the cell-intrinsic death machinery. Cell 91: 231-241, 1997.

24. Giri K and Aggarwal BB: Constitutive activation of NF-kappaB causes resistance to apoptosis in human cutaneous $\mathrm{T}$ cell lymphoma HuT-78 cells. Autocrine role of tumor necrosis factor and reactive oxygen intermediates. J Biol Chem 273 14008-14014, 1998.

25. Wang D and Dubois RN: The role of COX-2 in intestinal inflammation and colorectal cancer. Oncogene 29: 781-788, 2010.

26. Kirschenbaum A, Liu X, Yao S and Levine AC: The role of cyclooxygenase-2 in prostate cancer. Urology 58: 127-131, 2001.

27. Lee KS, Lee HJ, Ahn KS, et al: Cyclooxygenase-2/prostaglandin E2 pathway mediates icariside II induced apoptosis in human PC-3 prostate cancer cells. Cancer Lett 280: 93-100, 2009.

28. Hankey BF, Feuer EJ, Clegg LX, et al: Cancer surveillance series: Interpreting trends in prostate cancer-part I: evidence of the effects of screening in recent prostate cancer incidence, mortality, and survival rates. J Natl Cancer Inst 91: 1017-1024, 1999.

29. Ganesh B, Saoba SL, Sarade MN and Pinjari SV: Risk factors for prostate cancer: an hospital-based case-control study from Mumbai, India. Indian J Urol 27: 345-350, 2011.

30. American Dietetic Association; Dietitians of Canada: Position of the American Dietetic Association and Dietitians of Canada: Vegetarian diets. J Am Diet Assoc 103: 748-765, 2003.

31. Hsing AW and Chokkalingam AP: Prostate cancer epidemiology. Front Biosci 11: 1388-1413, 2006.

32. Rodrigues J, Abramjuk C, Vásquez L, et al: New 4-maleamic acid and 4-maleamide peptidyl chalcones as potential multitarget drugs for human prostate cancer. Pharm Res 28: 907-919, 2011.

33. Deb Majumdar I, Devanabanda A, Fox B, Schwartzman J, Cong H, Porco JA Jr, and Weber HC: Synthetic cyclohexenyl chalcone natural products possess cytotoxic activities against prostate cancer cells and inhibit cysteine cathepsins in vitro. Biochem Biophys Res Commun 416: 397-402, 2011.

34. Nagaraju M, Gnana Deepthi E, Ashwini C, et al: Synthesis and selective cytotoxic activity of novel hybrid chalcones against prostate cancer cells. Bioorg Med Chem Lett 22: 4314-4317, 2012.

35. Jung JI, Lim SS, Choi HJ, et al: Isoliquiritigenin induces apoptosis by depolarizing mitochondrial membranes in prostate cancer cells. J Nutr Biochem 17: 689-696, 2006.
36. Hengartner MO: The biochemistry of apoptosis. Nature 407: $770-776,2000$

37. Zamzami N, Susin SA, Marchetti P, et al: Mitochondrial control of nuclear apoptosis. J Exp Med 183: 1533-1544, 1996.

38. Green D and Kroemer G: The central executioners of apoptosis: caspases or mitochondria? Trends Cell Biol 8: 267-271, 1998

39. Liu X, Kim CN, Yang J, Jemmerson R and Wang X: Induction of apoptotic program in cell-free extracts: requirement for dATP and cytochrome c. Cell 86: 147-157, 1996.

40. Lebedeva IV, Sarkar D, Su ZZ, et al: Bcl-2 and Bcl-x(L) differentially protect human prostate cancer cells from induction of apoptosis by melanoma differentiation associated gene-7, mda-7/IL-24. Oncogene 22: 8758-8773, 2003.

41. Salvesen GS and Dixit VM: Caspases: intracellular signalling by proteolysis. Cell 91: 443-446, 1997.

42. D'Amours D, Desnoyers S, D'Silva I and Poirier GG: Poly(ADPribosyl)ation reactions in the regulation of nuclear functions. Biochem J 342: 249-268, 1999.

43. Nicholson DW: Caspase structure, proteolytic substrates, and function during apoptotic cell death. Cell Death Differ 6: 1028-1042, 1999.

44. Chen X, Thakkar H, Tyan F, et al: Constitutively active Akt is an important regulator of TRAIL sensitivity in prostate cancer. Oncogene 20: 6073-6083, 2001.

45. Murillo H, Huang H, Schmidt LJ, Smith DI and Tindall DJ: Role of PI3K signalling in survival and progression of LNCaP prostate cancer cells to the androgen refractory state. Endocrinology 142: 4795-4805, 2001.

46. Testa JR and Bellacosa A: AKT plays a central role in tumorigenesis. Proc Natl Acad Sci USA 98: 10983-10985, 2001.

47. Madrid LV, Mayo MW, Reuther JY and Baldwin AS Jr: Akt stimulates the transactivation potential of the RelA/p65 Subunit of NF-kappa B through utilization of the Ikappa B kinase and activation of the mitogen-activated protein kinase p38. J Biol Chem 276: 18934-18940, 2001.

48. Ahn KS, Sethi G and Aggarwal BB: Nuclear factor-kappa B: from clone to clinic. Curr Mol Med 7: 619-637, 2007.

49. Yang P, Cartwright CA, Li J, et al: Arachidonic acid metabolism in human prostate cancer. Int J Oncol 41: 1495-1503, 2012.

50. Narayanan NK, Narayanan BA and Reddy BS: A combination of docosahexaenoic acid and celecoxib prevents prostate cancer cell growth in vitro and is associated with modulation of nuclear factor- $\mathrm{\kappa B}$, and steroid hormone receptors. Int J Oncol 26: 785-792, 2005

51. Sarveswaran S, Gautam SC and Ghosh J: Wedelolactone, a medicinal plant-derived coumestan, induces caspase-dependent apoptosis in prostate cancer cells via downregulation of $\mathrm{PKC \varepsilon}$ without inhibiting Akt. Int J Oncol 41: 2191-2199, 2012.

52. Patsos HA, Greenhough A, Hicks DJ, et al: The endogenous cannabinoid, anandamide, induces COX-2-dependent cell death in apoptosis-resistant colon cancer cells. Int J Oncol 37: 187-193, 2010.

53. Limami Y, Pinon A, Leger DY, et al: HT-29 colorectal cancer cells undergoing apoptosis overexpress COX-2 to delay ursolic acid-induced cell death. Biochimie 93: 749-757, 2011.

54. Limami Y, Pinon A, Leger DY, et al: The P2Y2/Src/p38/COX-2 pathway is involved in the resistance to ursolic acid-induced apoptosis in colorectal and prostate cancer cells. Biochimie 94: 1754-1763, 2012.

55. Mencher SK and Wang LG: Promiscuous drugs compared to selective drugs (promiscuity can be a virtue). BMC Clin Pharmacol 5: 3, 2005.

56. Trouillas $\mathrm{P}$, Corbière $\mathrm{C}$, Liagre $\mathrm{B}$, Duroux JL and Beneytout JL: Structure-function relationship for saponin effects on cell cycle arrest and apoptosis in the human 1547 osteosarcoma cells: a molecular modelling approach of natural molecules structurally close to diosgenin. Bioorg Med Chem 13: 1141-1149, 2005 . 